Karl-Franzens Universität Graz

Technische Universität Graz

Medizinische Universität Graz

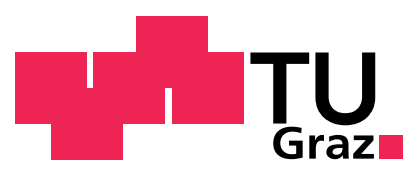

\title{
A measure space approach to optimal source placement
}

\author{
Christian Clason Karl Kunisch
}


SFB sponsors:

- Austrian Science Fund (FWF)

- University of Graz

- Graz University of Technology

- Medical University of Graz

- Government of Styria

- City of Graz

Das Land

Steiermark 


\title{
A MEASURE SPACE APPROACH TO OPTIMAL SOURCE PLACEMENT*
}

\author{
Christian Clason $^{\dagger} \quad$ Karl Kunisch ${ }^{\dagger}$
}

June 22, 2011

The problem of optimal placement of point sources is formulated as a distributed optimal control problem with sparsity constraints. For practical relevance, partial observations as well as partial and non-negative controls need to be considered. Although well-posedness of this problem requires a non-reflexive Banach space setting, a primal-predual formulation of the optimality system can be approximated well by a family of semi-smooth equations, which can be solved by a superlinearly convergent semi-smooth Newton method. Numerical examples indicate the feasibility for optimal light source placement problems in diffusive photochemotherapy.

\section{INTRODUCTION}

This work is concerned with the optimal control problem

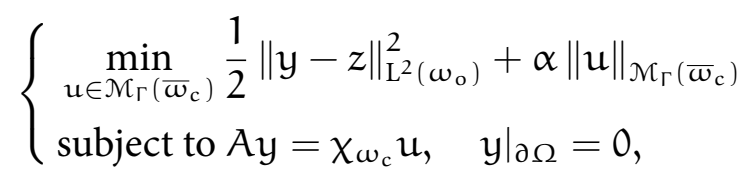

where $A$ is a linear second-order elliptic operator, $\omega_{0}$ and $\omega_{c}$ represent the observation and control subdomains of the bounded domain $\Omega \subset \mathbb{R}^{n}$ with characteristic function $\chi$, and $z \in \mathrm{L}^{2}\left(\omega_{\mathrm{o}}\right)$ is given. For convenience, we abbreviate $\Gamma=\partial \Omega$. Furthermore $\mathcal{M}_{\Gamma}\left(\bar{\omega}_{\mathrm{c}}\right)$

\footnotetext{
*This work was supported by the Austrian Science Fund (FWF) under grant SFB F32 (SFB "Mathematical Optimization and Applications in Biomedical Sciences").

${ }^{\dagger}$ Institute for Mathematics and Scientific Computing, University of Graz, Heinrichstrasse 36, A-8010 Graz, Austria, (christian.clason@uni-graz.at, karl.kunisch@uni-graz.at).
} 
denotes the topological dual of $\mathrm{C}_{\Gamma}\left(\bar{\omega}_{\mathrm{c}}\right):=\left\{v \in \mathrm{C}\left(\bar{\omega}_{\mathrm{c}}\right):\left.v\right|_{\partial \omega_{\mathrm{c}} \cap \Gamma}=0\right\}$, where the constraint $\left.v\right|_{\partial \omega_{c} \cap \Gamma}=0$ is dropped if $\partial \omega_{c} \cap \Gamma=\emptyset$. The norm on $\mathcal{M}_{\Gamma}\left(\bar{\omega}_{c}\right)$ is given by

$$
\|u\|_{\mathcal{M}_{\Gamma}\left(\bar{\omega}_{c}\right)}=\sup _{\substack{\varphi \in C_{\Gamma}\left(\bar{\omega}_{c}\right) \\\|\varphi\|_{C_{\Gamma}\left(\bar{\omega}_{\mathcal{c}}\right)} \leqslant 1}} \int_{\omega_{\mathcal{c}}} \varphi d u
$$

which coincides with $\|u\|_{L^{1}\left(\omega_{c}\right)}$ if $u \in L^{1}\left(\omega_{c}\right)$ (identified with a subspace of $\mathcal{M}_{\Gamma}\left(\bar{\omega}_{c}\right)$ ) holds. Since $\bar{\omega}_{c} \backslash \Gamma$ is a locally compact Hausdorff space, the Riesz representation theorem allows identifying elements of $\mathcal{M}_{\Gamma}\left(\bar{\omega}_{c}\right)$ with Radon measures that have compact support in $\bar{\omega}_{c} \backslash \Gamma$ (cf. [9, Th. VIII.2.19]).

The problem is motivated by the question of optimal source placement, e.g., in diffusive optical tomography, since the $\mathrm{L}^{1}$ norm is known to promote sparsity in optimization. The connection between $\mathrm{L}^{1}$ control costs and source placement was first discussed in [15]. However, problem $(\mathcal{P})$ is not well-posed in $\mathrm{L}^{1}$, since $\mathrm{L}^{1}$ lacks the necessary weak compactness properties. Problems with $\mathrm{L}^{1}$ control cost and $\mathrm{L}^{\infty}$ control constraints were considered in [15], [18], [19] and [5], while a measure space setting was first investigated in [6].

In this work, we address the feasibility of optimal source placement by optimal control in measure spaces by including partial observation, control on subdomains and non-negativity of the controls, which was not considered in the previously cited works. The Fenchel predual framework as utilized in [6] is not applicable in this situation, so we consider a primal-predual setting. This framework can be modified to allow for nonlinear control-to-state mappings, which also do not fit into the earlier Fenchel duality framework.

This paper is organized as follows. In section 2, we discuss the well-posedness of the optimal control problem for measure source terms defined on subdomains and derive the optimality system. Section 3 is devoted to the regularization of the optimality system and addresses the convergence of the regularized solutions to those of the original problem. The numerical solution using a semi-smooth Newton method is discussed in section 4. Finally, in section 5 we give numerical examples to indicate the feasibility of the proposed approach for a problem of optimal light source placement in photochemotherapy.

Throughout, we take as $\mathrm{W}_{0}^{1, \mathrm{r}}(\Omega)$ the closure of $\left\{v \in \mathrm{C}^{\infty}(\Omega):\left.v\right|_{\partial \Omega}=0\right\}$ in the $\mathrm{W}^{1, \mathrm{r}}(\Omega)$ norm, $r \in(1, \infty)$. We denote by $\mathrm{W}^{-1, \mathrm{r}^{\prime}}(\Omega)=\left(\mathrm{W}_{0}^{1, \mathrm{r}}(\Omega)\right)^{*}$ the topological dual of $\mathrm{W}_{0}^{1, \mathrm{r}}(\Omega)$. Moreover, for $\omega \subset \Omega$ we set $\mathrm{W}^{1, \mathrm{r}}(\omega)=\left\{\left.\varphi\right|_{\omega}: \varphi \in \mathrm{W}_{0}^{1, \mathrm{r}}(\Omega)\right\}$ with dual denoted by $\left(\mathrm{W}^{1, \mathrm{r}}(\omega)\right)^{*}$.

\section{PROBLEM FORMULATION AND OPTIMALITY SYSTEM}

We first address the well-posedness of the state equation. Let $\mathcal{M}(\Omega)$ denote the topological dual of $\mathrm{C}_{0}(\bar{\Omega})$ endowed with the operator norm, cf. (1.1). By the Riesz representation theorem (e.g., [9, Th. VIII.2.10]), $\mathcal{M}(\Omega)$ can be identified with the Banach space of finite Radon measures. We further choose $\mathrm{q} \in\left(1, \frac{n}{n-1}\right)$ and set $\mathrm{q}^{\prime}=\frac{\mathrm{q}-1}{\mathrm{q}} \in(\mathrm{n}, \infty)$. For this choice, we have $\mathrm{W}_{0}^{1, \mathrm{q}^{\prime}}(\Omega) \hookrightarrow \mathrm{C}_{0}(\bar{\Omega})$, and this embedding is compact. 
We consider the operator

$$
A y=-\sum_{j, k=1}^{n} \partial_{j}\left(a_{j k}(x) \partial_{k} y+d_{j}(x) y\right)+\sum_{j=1}^{n} b_{j}(x) \partial_{j} y+d(x) y,
$$

and for $\mu \in \mathcal{M}(\Omega)$ the abstract Dirichlet problem

$$
\left\{\begin{aligned}
A y=\mu, & \text { in } \Omega, \\
y=0, & \text { on } \partial \Omega,
\end{aligned}\right.
$$

which is to be interpreted in variational form, i.e., $y$ satisfies

$$
\text { (2.2) }-\sum_{j, k=1}^{n}\left\langle a_{j k} \partial_{j} y, \partial_{k} v\right\rangle_{L^{2}}+\sum_{j=1}^{n}\left\langle b_{j} \partial_{j} y, v\right\rangle_{L^{2}}+\sum_{k=1}^{n}\left\langle y, d_{k} \partial_{k} v\right\rangle_{L^{2}}+\langle d y, v\rangle_{L^{2}}=\int_{\Omega} v d \mu
$$

for all $v \in \mathrm{W}_{0}^{1, \mathrm{q}^{\prime}}(\Omega)$. Here, $\Omega$ is a bounded domain in $\mathbb{R}^{n}$ with $C^{1, \delta}$ boundary $\partial \Omega, \mathrm{a}_{\mathrm{jk}}, \mathrm{b}_{\mathrm{j}} \in$ $\mathrm{C}^{0, \delta}(\bar{\Omega})$ for some $\delta \in(0,1), d_{j}, d \in L^{\infty}(\Omega)$, and it is assumed that 0 is not an eigenvalue of $A$. These assumptions imply that the adjoint $A^{*}$ of $A$ is an isomorphism from $W_{0}^{1, q^{\prime}}(\Omega)$ to $\mathrm{W}^{-1, \mathrm{q}^{\prime}}(\Omega)$, see, e.g., [17, Th. 3.16], [10]. Consequently, $A$ is an isomorphism from $\mathrm{W}_{0}^{1, q}(\Omega)$ to $\mathrm{W}^{-1, \mathrm{q}}(\Omega)$. In particular, $(2.1)$ admits a unique solution satisfying

$$
\|y\|_{\mathrm{W}_{\mathrm{o}}^{1, \mathrm{q}}(\Omega)} \leqslant \mathrm{C}\|\mu\|_{\mathcal{M}(\Omega)}
$$

for a constant $C$ independent of $\mu$, by the fact that $\mathcal{M}(\Omega)$ embeds continuously into $\mathrm{W}^{-1, \mathrm{q}}(\Omega)$ (see, e.g., [16, Th. 9.1] and [14, Th. 4.1]). We refer to [14] for a discussion of the various (equivalent) characterizations of solutions to (2.2) and their uniqueness if $A^{*}$ is not surjective on $\mathrm{W}^{-1, \mathrm{q}^{\prime}}(\Omega)$.

We now define the control-to-state mapping associated to $(\mathcal{P})$. For this purpose, let

$$
\mathrm{R}_{\omega_{\mathrm{o}}}: \mathrm{W}_{0}^{1, \mathrm{q}}(\Omega) \rightarrow \mathrm{W}^{1, \mathrm{q}}\left(\omega_{\mathrm{o}}\right), \quad \mathrm{R}_{\omega_{\mathrm{c}}}: \mathrm{W}_{0}^{1, \mathrm{q}^{\prime}}(\Omega) \rightarrow \mathrm{W}^{1, \mathrm{q}^{\prime}}\left(\omega_{\mathrm{c}}\right)
$$

denote the canonical restriction operators from $\Omega$ to $\omega_{c}$ and $\omega_{\mathrm{o}}$, respectively, with adjoints

$$
\mathrm{R}_{\omega_{\mathrm{o}}}^{*}:\left(\mathrm{W}^{1, \mathrm{q}}\left(\omega_{\mathrm{o}}\right)\right)^{*} \rightarrow \mathrm{W}^{-1, \mathrm{q}^{\prime}}(\Omega), \quad \mathrm{R}_{\omega_{\mathrm{c}}}^{*}:\left(\mathrm{W}^{1, \mathrm{q}^{\prime}}\left(\omega_{\mathrm{c}}\right)\right)^{*} \rightarrow \mathrm{W}^{-1, \mathrm{q}}(\Omega) .
$$

Further we shall employ the injections

$$
\mathcal{J}: \mathrm{W}^{1, \mathrm{q}}\left(\omega_{\mathrm{o}}\right) \rightarrow \mathrm{L}^{2}\left(\omega_{\mathrm{o}}\right), \quad \mathcal{J}: \mathrm{W}^{1, \mathrm{q}^{\prime}}\left(\omega_{\mathrm{c}}\right) \rightarrow \mathrm{C}_{\Gamma}\left(\bar{\omega}_{\mathrm{c}}\right)
$$

with adjoints

$$
\mathcal{J}^{*}: \mathrm{L}^{2}\left(\omega_{\mathrm{o}}\right) \rightarrow\left(\mathrm{W}^{1, \mathrm{q}}\left(\omega_{\mathrm{o}}\right)\right)^{*}, \quad \mathcal{J}^{*}: \mathcal{M}_{\Gamma}\left(\bar{\omega}_{\mathrm{c}}\right) \rightarrow\left(\mathrm{W}^{1, \mathrm{q}^{\prime}}\left(\omega_{\mathrm{c}}\right)\right)^{*} .
$$

Then we set

$$
\mathrm{S}_{\omega}: \mathcal{M}_{\Gamma}\left(\bar{\omega}_{\mathrm{c}}\right) \rightarrow \mathrm{L}^{2}\left(\omega_{\mathrm{o}}\right), \quad \mathrm{u} \mapsto \mathcal{J R}_{\omega_{\mathrm{o}}} A^{-1} \mathrm{R}_{\omega_{\mathrm{c}}}^{*} \mathcal{J}^{*} u
$$


and note that $S_{\omega}$ is a bounded linear operator. Since $R_{\omega_{0}}, R_{\omega_{c}}$, J and $\mathcal{J}$ have dense ranges, their adjoints are injections. To argue that $\mathcal{J}$ has dense range, let $\varphi \in \mathrm{C}_{\Gamma}\left(\bar{\omega}_{\mathrm{c}}\right)$. By Tietze's extension theorem (e.g., [7, Th. 3.1]), there exists a $\widetilde{\varphi} \in \mathrm{C}_{\Gamma}\left(\bar{\omega}_{\mathrm{c}}\right)$ with $\left.\widetilde{\varphi}\right|_{\partial \Omega}=0$ and $\left.\widetilde{\varphi}\right|_{\bar{\omega}_{\mathrm{c}}}=\varphi$ (see also $\left[17\right.$, Th. 1.N]). Moreover $\widetilde{\varphi}$ can be approximated by $\widetilde{\varphi}_{n} \in \mathrm{W}_{0}^{1, \mathrm{q}^{\prime}}(\Omega)$ in the $\mathrm{W}_{0}^{1, \mathrm{q}^{\prime}}(\Omega)$-norm, and hence $\left.\widetilde{\varphi}_{n}\right|_{\bar{\omega}_{\mathrm{c}}} \in \mathrm{W}^{1, \mathrm{q}^{\prime}}\left(\omega_{\mathrm{c}}\right)$ approximates $\varphi \in \mathrm{C}_{\Gamma}\left(\bar{\omega}_{\mathrm{c}}\right)$.

We will also need the following continuity property of the control-to-state mapping.

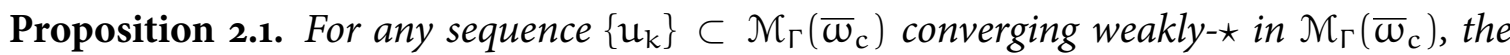
sequence $\mathrm{S}_{\omega}\left(\mathrm{u}_{\mathrm{k}}\right)$ converges strongly to $\mathrm{S}_{\omega}(\mathrm{u})$ in $\mathrm{L}^{2}\left(\omega_{\mathrm{o}}\right)$.

Proof. Since $\mathrm{q}^{\prime}>\mathrm{n}$, the embedding $\mathrm{W}^{1, \mathrm{q}^{\prime}}\left(\omega_{\mathrm{c}}\right) \hookrightarrow \mathrm{C}_{\Gamma}\left(\bar{\omega}_{\mathrm{c}}\right)$ is compact. Therefore, the adjoint embedding $\mathcal{M}_{\Gamma}\left(\bar{\omega}_{\mathrm{c}}\right) \hookrightarrow\left(\mathrm{W}^{1, \mathrm{q}^{\prime}}\left(\omega_{\mathrm{c}}\right)\right)^{*}$ is compact as well. Weak- $\star$ convergence of $\mathfrak{u}_{k}$ in $\mathcal{M}_{\Gamma}\left(\bar{\omega}_{c}\right)$ thus implies strong convergence of $\mathcal{J}^{*} \mathfrak{u}_{k}$. The claim then follows from the continuity of $\mathcal{J} R_{\omega_{0}} A^{-1} R_{\omega_{c}}^{*}$.

The reduced problem corresponding to $(\mathcal{P})$ can then be formulated as

$$
\min _{u \in \mathcal{M}_{\Gamma}\left(\bar{\omega}_{\mathfrak{c}}\right)} \frac{1}{2}\left\|S_{\omega} u-z\right\|_{L^{2}\left(\omega_{\mathrm{o}}\right)}^{2}+\alpha\|u\|_{\mathcal{M}_{\Gamma}\left(\bar{\omega}_{\mathfrak{c}}\right)} .
$$

Existence of a minimizer $u^{*}$ follows from the fact that bounded sequences in $\mathcal{M}_{\Gamma}\left(\bar{\omega}_{\mathrm{c}}\right)$ contain a weakly- $\star$ convergent subsequence, and that $u \mapsto\|u\|_{\mathcal{M}_{\Gamma}\left(\bar{\omega}_{c}\right)}$ is weak- $\star$ lower semicontinuous. Remark 2.2. If a minimizer $u^{*}$ satisfies $u^{*} \in L^{1}\left(\omega_{c}\right)$, it is also a solution of the problem

$$
\min _{u \in L^{1}\left(\omega_{0}\right)} \frac{1}{2}\left\|S_{\omega} u-z\right\|_{L^{2}\left(\omega_{0}\right)}^{2}+\alpha\|u\|_{L^{1}\left(\omega_{c}\right)} .
$$

This follows from the embedding of $\mathrm{L}^{1}\left(\omega_{\mathrm{c}}\right)$ into $\mathcal{M}_{\Gamma}\left(\bar{\omega}_{\mathrm{c}}\right)$ and the fact that $\|v\|_{\mathcal{M}_{\Gamma}\left(\bar{\omega}_{\mathrm{c}}\right)}=$ $\|v\|_{\mathrm{L}^{1}\left(\omega_{\mathrm{c}}\right)}$ for $v \in \mathrm{L}^{1}\left(\omega_{\mathrm{c}}\right)$ (cf. [4, Ch. IV]).

To derive a necessary optimality condition for (2.3), we shall utilize the operator

$$
{ }^{*} \mathrm{~S}_{\omega}: \mathrm{L}^{2}\left(\omega_{\mathrm{o}}\right) \rightarrow \mathrm{C}_{\Gamma}\left(\bar{\omega}_{\mathrm{c}}\right), \quad \varphi \mapsto \mathcal{J} \mathrm{R}_{\omega_{\mathrm{c}}}^{*}\left(A^{*}\right)^{-1} \mathrm{R}_{\omega_{\mathrm{o}}} \mathcal{J}^{*} \varphi,
$$

noting that

$$
\left({ }^{*} S_{\omega}\right)^{*}=S_{\omega},
$$

i. e., ${ }^{*} S_{\omega}$ is the "preadjoint" to $S_{\omega}$.

Theorem 2.3. Let $\mathrm{u}^{*} \in \mathcal{M}_{\Gamma}\left(\bar{\omega}_{\mathrm{c}}\right)$ be a solution to (2.3). Then there exists a $\mathrm{p}^{*} \in \mathrm{C}_{\Gamma}\left(\bar{\omega}_{\mathrm{c}}\right)$ satisfying

$$
\left\{\begin{aligned}
{ }^{*} S_{\omega}\left(S_{\omega} u^{*}-z\right) & =p^{*}, \\
\left\langle u^{*}, p^{*}-p\right\rangle_{\mathcal{M}_{\Gamma}\left(\bar{\omega}_{c}\right), C_{\Gamma}\left(\bar{\omega}_{c}\right)} & \leqslant 0, \quad\left\|p^{*}\right\|_{C_{\Gamma}\left(\bar{\omega}_{c}\right)} \leqslant \alpha,
\end{aligned}\right.
$$

for all $\mathrm{p} \in \mathrm{C}_{\Gamma}\left(\bar{\omega}_{\mathrm{c}}\right)$ with $\|\mathrm{p}\|_{\mathrm{C}_{\Gamma}\left(\bar{\omega}_{\mathrm{c}}\right)} \leqslant \alpha$. 
Proof. We shall employ a Fenchel duality argument. To avoid dealing with $\left(\mathcal{M}_{\Gamma}\left(\bar{\omega}_{\mathrm{c}}\right)\right)^{*}$, we shall consider a predual of (2.3) rather than a dual problem. Such a procedure was previously used in $[3,6,11]$, for example. For this purpose we consider the following problem in $\mathrm{L}^{2}\left(\omega_{\mathrm{o}}\right)$ :

$$
\begin{array}{r}
\min _{\mathbf{q} \in \mathrm{L}^{2}\left(\omega_{o}\right)} \frac{1}{2}\|\mathrm{q}+z\|_{\mathrm{L}^{2}\left(\omega_{\mathrm{o}}\right)}^{2}-\frac{1}{2}\|z\|_{\mathrm{L}^{2}\left(\omega_{\mathrm{o}}\right)}^{2}+\mathrm{I}_{\left\{\|\mathbf{q}\|_{\mathrm{C}_{\Gamma}\left(\bar{\omega}_{\mathcal{c}}\right)} \leqslant \alpha\right\}}\left({ }^{*} S_{\omega} \mathrm{q}\right) \\
=: \min _{\mathbf{q} \in \mathrm{L}^{2}\left(\omega_{\mathrm{o}}\right)} \mathcal{F}(\mathbf{q})+\mathcal{G}\left({ }^{*} S_{\omega} \mathbf{q}\right),
\end{array}
$$

where $\mathcal{F}: \mathrm{L}^{2}\left(\omega_{\mathrm{o}}\right) \rightarrow \mathbb{R}$ and $\mathcal{G}: \mathrm{C}_{\Gamma}\left(\bar{\omega}_{\mathrm{c}}\right) \rightarrow \mathbb{R} \cup\{\infty\}$. A short computation shows that the Fenchel conjugates $\mathcal{F}^{*}: \mathrm{L}^{2}\left(\omega_{\mathrm{o}}\right) \rightarrow \mathbb{R}$ and $\mathcal{G}^{*}: \mathcal{M}_{\Gamma}\left(\bar{\omega}_{\mathrm{c}}\right) \rightarrow \mathbb{R}$ are given by

$$
\mathcal{F}^{*}(v)=\frac{1}{2}\|v-z\|_{\mathrm{L}^{2}\left(\omega_{\mathrm{o}}\right)}^{2}, \quad \mathcal{G}^{*}(v)=\alpha\|v\|_{\mathcal{M}_{\Gamma}\left(\bar{\omega}_{\mathrm{c}}\right)} .
$$

Since $\mathrm{q} \mapsto \mathcal{F}(\mathrm{q})+\mathcal{G}\left({ }^{*} S_{\omega} \mathbf{q}\right)$ is continuous at 0 , the Fenchel duality theorem (see, e. g., [8, Th. 4.1]) is applicable and implies that

$$
\begin{aligned}
\min _{\mathbf{q} \in \mathrm{L}^{2}\left(\omega_{o}\right)} \mathcal{F}(\mathbf{q})+\mathcal{G}\left({ }^{*} S_{\omega} \mathbf{q}\right) & =\min _{\mathfrak{u} \in \mathcal{M}_{\Gamma}\left(\bar{\omega}_{c}\right)} \mathcal{F}^{*}\left(S_{\omega} u\right)+\mathcal{G}^{*}(-\mathfrak{u}) \\
& =\min _{\mathfrak{u} \in \mathcal{M}_{\Gamma}\left(\bar{\omega}_{c}\right)} \frac{1}{2}\left\|S_{\omega} u-z\right\|_{\mathrm{L}^{2}\left(\omega_{o}\right)}^{2}+\alpha\|u\|_{\mathcal{M}_{\Gamma}\left(\bar{\omega}_{c}\right)},
\end{aligned}
$$

where we utilize $\left({ }^{*} S_{\omega}\right)^{*}=S_{\omega}$. Moreover (cf. [8, Prop. 4.1]), to every minimizer $q^{*} \in L^{2}\left(\omega_{o}\right)$ of the left hand side of (2.5) corresponds a minimizer $u^{*} \in \mathcal{M}_{\Gamma}\left(\bar{\omega}_{c}\right)$ of the right hand side satisfying the relationship

$$
\left\{\begin{aligned}
S_{\omega} u^{*} & =q^{*}+z, \\
-u^{*} & \in \partial I_{\left\{\|q\|_{C_{\Gamma}\left(\bar{\omega}_{\mathcal{c}}\right)} \leqslant \alpha\right\}}\left({ }^{*} S_{\omega} q^{*}\right) .
\end{aligned}\right.
$$

From the second relation, we have $\left\|{ }^{*} S_{\omega} q^{*}\right\|_{C_{\Gamma}\left(\bar{\omega}_{c}\right)} \leqslant \alpha$ and

$$
\left\langle-u^{*}, p-{ }^{*} S_{\omega} q^{*}\right\rangle_{\mathcal{M}_{\Gamma}\left(\bar{\omega}_{c}\right), C_{\Gamma}\left(\bar{\omega}_{c}\right)} \leqslant 0 \quad \text { for all }\|p\|_{C_{\Gamma}\left(\bar{\omega}_{c}\right)} \leqslant \alpha .
$$

Setting $\mathrm{p}^{*}={ }^{*} S_{\omega} \mathrm{q}^{*}={ }^{*} S_{\omega}\left(S_{\omega} \mathrm{u}^{*}-z\right)$ we find that

$$
\left\langle u^{*}, p^{*}-p\right\rangle_{\mathcal{M}_{\Gamma}\left(\bar{\omega}_{c}\right), C_{\Gamma}\left(\bar{\omega}_{c}\right)} \leqslant 0 \quad \text { for all }\|p\|_{\mathrm{C}_{\Gamma}\left(\bar{\omega}_{c}\right)} \leqslant \alpha
$$

and $\left\|p^{*}\right\|_{C_{\Gamma}\left(\bar{\omega}_{c}\right)} \leqslant \alpha$.

We note that by construction $\mathrm{p}^{*} \in \mathrm{W}^{1, \mathrm{q}^{\prime}}\left(\omega_{\mathrm{c}}\right)$ holds. From the second relation of (OS), we can also obtain the following structural information on an optimal control $u^{*}$.

Corollary 2.4. Let $\left(\mathrm{u}^{*}, \mathrm{p}^{*}\right)$ be a solution to (OS). Then for any $\mathrm{p} \in \mathrm{C}_{\Gamma}\left(\overline{\boldsymbol{\omega}}_{\mathrm{c}}\right)$ with $\mathrm{p} \geqslant 0$,

$$
\begin{array}{ll}
\left\langle u^{*}, p\right\rangle_{\mathcal{M}_{\Gamma}\left(\bar{\omega}_{c}\right), C_{\Gamma}\left(\bar{\omega}_{c}\right)=0} & \text { if } \operatorname{supp}(p) \subset\left\{x \in \bar{\omega}_{c}:\left|p^{*}(x)\right|<\alpha\right\}, \\
\left\langle u^{*}, p\right\rangle_{\mathcal{M}_{\Gamma}\left(\bar{\omega}_{c}\right), C_{\Gamma}\left(\bar{\omega}_{c}\right)} \leqslant 0 & \text { if } \operatorname{supp}(p) \subset\left\{x \in \bar{\omega}_{c}: p^{*}(x)=\alpha\right\}, \\
\left\langle u^{*}, p\right\rangle_{\mathcal{M}_{\Gamma}\left(\bar{\omega}_{c}\right), C_{\Gamma}\left(\bar{\omega}_{c}\right)} \geqslant 0 & \text { if } \operatorname{supp}(p) \subset\left\{x \in \bar{\omega}_{c}: p^{*}(x)=-\alpha\right\} .
\end{array}
$$


This can be interpreted as a sparsity property: An optimal control $u^{*}$ will be non-zero only on sets where the constraint on $p^{*}$ is active; hence the larger the penalty $\alpha$, the smaller the support of the control.

Remark 2.5 (Non-negative controls). If in $(\mathcal{P})$ only non-negative controls are admitted, we replace $\mathcal{G}^{*}(v)$ by

$$
\mathcal{G}_{+}^{*}: \mathcal{M}_{\Gamma}\left(\bar{\omega}_{\mathrm{c}}\right) \rightarrow \mathbb{R} \cup\{\infty\}, \quad v \mapsto \mathrm{I}_{\{v \leqslant 0\}}+\alpha\|v\|_{\mathcal{M}_{\Gamma}\left(\bar{\omega}_{\mathrm{c}}\right)}
$$

(noting that the dual problem involves the term $\mathcal{G}_{+}^{*}\left(-u^{*}\right)$ ). This is the Fenchel dual of

$$
\mathcal{G}_{+}: \mathrm{C}_{\Gamma}\left(\bar{\omega}_{\mathrm{c}}\right) \rightarrow \mathbb{R} \cup\{\infty\}, \quad \mathrm{q} \mapsto \mathrm{I}_{\{\mathrm{q} \geqslant-\alpha\}},
$$

and (2.6) must be replaced by

$$
\left\langle-u^{*}, p-{ }^{*} S_{\omega} q^{*}\right\rangle_{\mathcal{M}_{\Gamma}\left(\bar{\omega}_{c}\right), C_{\Gamma}\left(\bar{\omega}_{c}\right)} \leqslant 0 \quad \text { for all } p \geqslant-\alpha .
$$

The optimality conditions for the case of non-negative controls become

$\left(\mathrm{OS}_{+}\right)$

$$
\left\{\begin{array}{c}
{ }^{*} S_{\omega}\left(S_{\omega} u^{*}-z\right)=p^{*}, \\
\left\langle u^{*}, p^{*}-p\right\rangle_{\mathcal{M}_{\Gamma}\left(\bar{\omega}_{c}\right), C_{\Gamma}\left(\bar{\omega}_{c}\right)} \leqslant 0, \quad p^{*} \geqslant-\alpha
\end{array}\right.
$$

for all $p \in \mathrm{C}_{\Gamma}\left(\bar{\omega}_{\mathrm{c}}\right)$ with $p \geqslant-\alpha$.

\section{REGULARIZATION}

The numerical solution of the optimality system (OS) is based on a Moreau-Yoshida regularization of (OS). For given $c>0$, we search for $\left(u_{c}, p_{c}\right) \in L^{2}\left(\omega_{c}\right) \times W^{1, q^{\prime}}\left(\omega_{c}\right)$ which satisfy

$$
\left\{\begin{aligned}
p_{c} & =S_{\omega}^{*}\left(S_{\omega} u_{c}-z\right), \\
-u_{c} & =c \max \left(0, p_{c}-\alpha\right)+c \min \left(0, p_{c}+\alpha\right),
\end{aligned}\right.
$$

where the max and min are taken pointwise in $\bar{\omega}_{c}$. Here, $S_{\omega}$ is considered as an operator from $\mathrm{L}^{2}\left(\omega_{\mathrm{c}}\right) \rightarrow \mathrm{L}^{2}\left(\omega_{\mathrm{o}}\right)$. The action of its adjoint $\mathrm{S}_{\omega}^{*}: \mathrm{L}^{2}\left(\omega_{\mathrm{o}}\right) \rightarrow \mathrm{L}^{2}\left(\omega_{\mathrm{c}}\right)$ coincides with that of ${ }^{*} S_{\omega}$. Moreover, the range of $S_{\omega}^{*}$ is contained in $W^{1, q^{\prime}}\left(\omega_{c}\right)$.

This regularization can be interpreted as a quadratic penalization of the box constraints in (2.4).

Theorem 3.1. There exists a unique solution $\left(\mathrm{u}_{\mathrm{c}}, \mathrm{p}_{\mathrm{c}}\right) \in \mathrm{L}^{2}\left(\omega_{\mathrm{c}}\right) \times \mathrm{W}^{1, \mathrm{q}^{\prime}}\left(\omega_{\mathrm{c}}\right)$ of $\left(\mathrm{OS}_{\mathrm{c}}\right)$.

Proof. The claim will follow from the fact that $\left(\mathrm{OS}_{\mathrm{c}}\right)$ are the necessary optimality conditions of the problem

$$
\min _{u \in \mathrm{L}^{2}\left(\omega_{c}\right)} \frac{1}{2}\left\|S_{\omega} u-z\right\|_{L^{2}\left(\omega_{o}\right)}^{2}+\alpha\|u\|_{L^{1}\left(\omega_{c}\right)}+\frac{1}{2 c}\|u\|_{L^{2}\left(\omega_{c}\right)}^{2} .
$$


The cost function in $\left(\mathcal{P}_{c}\right)$ is continuous, bounded from below and strictly convex due to the presence of the $\mathrm{L}^{2}\left(\omega_{c}\right)$ term, hence $\left(\mathcal{P}_{c}\right)$ admits a unique minimizer $u_{c} \in \mathrm{L}^{2}\left(\omega_{c}\right)$. To express $\left(\mathcal{P}_{c}\right)$ abstractly, we introduce

$$
\begin{aligned}
& \mathcal{F}_{\mathrm{c}}^{*}: \mathrm{L}^{2}\left(\omega_{\mathrm{c}}\right) \rightarrow \mathbb{R}, \quad \mathrm{u} \mapsto \frac{1}{2}\left\|S_{\omega} \mathrm{u}-z\right\|_{\mathrm{L}^{2}\left(\omega_{\mathrm{o}}\right)}^{2}, \\
& \mathcal{G}_{\mathrm{c}}^{*}: \mathrm{L}^{2}\left(\omega_{\mathrm{c}}\right) \rightarrow \mathbb{R}, \quad \mathrm{u} \mapsto \alpha\|\mathrm{u}\|_{\mathrm{L}^{1}\left(\omega_{\mathrm{c}}\right)}+\frac{1}{2 \mathrm{c}}\|\mathrm{u}\|_{\mathrm{L}^{2}\left(\omega_{\mathrm{c}}\right)}^{2} .
\end{aligned}
$$

The optimality condition for $\left(\mathcal{P}_{c}\right)$ is given by

$$
0 \in S_{\omega}^{*}\left(S_{\omega} u_{c}-z\right)+\partial \mathcal{G}_{c}^{*}\left(u_{c}\right)
$$

or equivalently,

$$
\left\{\begin{aligned}
p_{c} & =S_{\omega}^{*}\left(S_{\omega} u_{c}-z\right), \\
-p_{c} & \in \partial \mathcal{G}_{c}^{*}\left(u_{c}\right)
\end{aligned}\right.
$$

where the first equation implies $p_{c} \in \mathrm{W}^{1, \mathrm{q}^{\prime}}\left(\omega_{\mathrm{c}}\right) \hookrightarrow \mathrm{C}_{\Gamma}\left(\bar{\omega}_{\mathrm{c}}\right)$.

We claim that $\mathcal{G}_{\mathrm{c}}^{*}$ is the Fenchel conjugate of

$$
\mathcal{G}_{c}: L^{2}\left(\omega_{c}\right) \rightarrow \mathbb{R}, \quad p \mapsto \frac{c}{2}\|\max (0, p-\alpha)\|_{L^{2}\left(\omega_{c}\right)}^{2}+\frac{c}{2}\|\min (0, p+\alpha)\|_{L^{2}\left(\omega_{c}\right)}^{2} .
$$

To show this, we compute the Fenchel conjugate of $\mathcal{G}_{c}$ at $u \in \mathrm{L}^{2}\left(\omega_{c}\right)$, which is defined as

$$
\mathcal{G}_{c}^{*}(u)=\sup _{q \in \mathrm{L}^{2}\left(\omega_{c}\right)}\langle u, q\rangle_{L^{2}\left(\omega_{c}\right)}-\mathcal{G}_{c}(q) .
$$

The supremum is attained at $p \in \mathrm{L}^{2}\left(\omega_{\mathrm{c}}\right)$ if and only if

$$
u=\partial \mathcal{G}_{c}(p)=c \max (0, p-\alpha)+c \min (0, p+\alpha)
$$

holds almost everywhere in $\omega_{c}$. If $u(x)>0$, the right hand side has to be positive as well, which implies that $\mathfrak{u}(x)=\mathfrak{c}(p(x)-\alpha)$ and hence $p(x)=\frac{1}{c} \mathfrak{u}(x)+\alpha$. Similarly, $\mathfrak{u}(x)<0$ yields $p(x)=\frac{1}{c} u(x)-\alpha$. For $u(x)=0$, we deduce that $-\alpha \leqslant p(x) \leqslant \alpha$ holds. Substituting in the definition of $\mathcal{G}^{*}$, we have that

$$
\begin{aligned}
\mathcal{G}_{c}^{*}(u)= & \int_{\{u>0\}} u(x)\left(\frac{1}{c} u(x)+\alpha\right)-\frac{1}{2 c} \max (0, u(x))^{2} d x \\
& +\int_{\{u<0\}} u(x)\left(\frac{1}{c} u(x)-\alpha\right)-\frac{1}{2 c} \min (0, u(x))^{2} d x \\
= & \frac{1}{2 c}\|u\|_{L^{2}\left(\omega_{c}\right)}^{2}+\alpha\|u\|_{L^{1}\left(\omega_{c}\right)} .
\end{aligned}
$$

Since $\mathcal{G}_{c}$ is Lipschitz continuous, the second condition in (3.1) can be expressed as (cf., e. g., [1, Th. 9.5.1])

$$
u_{c} \in \partial \mathcal{G}_{c}\left(-p_{c}\right)=\left\{c\left(\max \left(0,-p_{c}-\alpha\right)+\min \left(0,-p_{c}+\alpha\right)\right)\right\} .
$$


Noting that $\max (0,-p)=-\min (0, p)$, the optimality conditions $\left(\operatorname{OS}_{c}\right)$ follow.

Turning to uniqueness, let $\left(u_{c}, p_{c}\right)$ and $\left(\bar{u}_{c}, \bar{p}_{c}\right)$ be two solutions to $\left(\operatorname{OS}_{c}\right)$ and set $(\delta u, \delta p)=$ $\left(u_{c}-\bar{u}_{c}, p_{c}-\bar{p}_{c}\right)$. Then, subtracting the corresponding optimality conditions and taking the inner product with $(\delta u, \delta p)$ implies that

$$
\begin{aligned}
0=\left\|S_{\omega} \delta u\right\|_{L^{2}\left(\omega_{o}\right)}^{2} & +c\left\langle\max \left(0, p_{c}-\alpha\right)-\max \left(0, \bar{p}_{c}-\alpha\right), p_{c}-\bar{p}_{c}\right\rangle_{L^{2}\left(\omega_{c}\right)} \cdot \\
& +c\left\langle\min \left(0, p_{c}+\alpha\right)-\min \left(0, \bar{p}_{c}+\alpha\right), p_{c}-\bar{p}_{c}\right\rangle_{L^{2}\left(\omega_{c}\right)}
\end{aligned}
$$

Since the mappings $p \mapsto \max (0, p)$ and $p \mapsto \min (0, p)$ are monotone, we obtain that the inner products in (3.2) are non-negative and thus that $S_{\omega} \delta u=0$. Since $\delta p=S_{\omega}^{*}\left(S_{\omega} \delta u\right)=0$ by linearity of state and adjoint equation, we deduce $p_{c}=\bar{p}_{c}$ and hence $u_{c}=\bar{u}_{c}$ from the second equation of $\left(\mathrm{OS}_{\mathrm{c}}\right)$.

Next, we address the convergence of solutions of $\left(\mathrm{OS}_{\mathrm{c}}\right)$ as $\mathrm{c} \rightarrow \infty$.

Theorem 3.2. Let $\left(u_{c}, p_{c}\right) \in \mathrm{L}^{2}\left(\omega_{c}\right) \times \mathrm{W}^{1, q^{\prime}}\left(\omega_{c}\right)$ be solutions of $\left(\mathrm{OS}_{\mathrm{c}}\right)$ for $\mathrm{c}>0$. Then the family $\left(\mathrm{u}_{\mathrm{c}}, \mathrm{p}_{\mathrm{c}}\right)$ contains a subsequence, denoted by the same symbol, such that

$$
\begin{aligned}
& \mathrm{u}_{\mathrm{c}} \rightarrow^{\star} \mathrm{u}^{*} \quad \text { in } \mathcal{M}_{\Gamma}\left(\bar{\omega}_{\mathrm{c}}\right), \\
& \mathrm{p}_{\mathrm{c}} \rightarrow \mathrm{p}^{*} \quad \text { in } \mathrm{W}^{1, \mathrm{q}^{\prime}}\left(\omega_{\mathrm{c}}\right) \text { and hence in } \mathrm{C}_{\Gamma}\left(\bar{\omega}_{\mathrm{c}}\right),
\end{aligned}
$$

and $\left(\mathrm{u}^{*}, \mathrm{p}^{*}\right)$ is a solution of $(\mathrm{OS})$.

Proof. Since $u_{c}=0$ is an admissible control, we have

$$
\begin{aligned}
\alpha\left\|u_{c}\right\|_{\mathcal{M}_{\Gamma}\left(\bar{\omega}_{c}\right)} & \leqslant \frac{1}{2}\left\|S_{\omega} u_{c}-z\right\|_{L^{2}\left(\omega_{o}\right)}^{2}+\alpha\left\|u_{c}\right\|_{\mathcal{M}_{\Gamma}\left(\bar{\omega}_{c}\right)}+\frac{1}{2 c}\left\|u_{c}\right\|_{L^{2}\left(\omega_{c}\right)}^{2} \\
& \leqslant \frac{1}{2}\|z\|_{L^{2}\left(\omega_{o}\right)}^{2} .
\end{aligned}
$$

The family of minimizers $\left\{u_{c}\right\}_{\mathcal{c}>0}$ is thus bounded in $\mathcal{M}_{\Gamma}\left(\bar{\omega}_{c}\right)$, and hence there exists a subsequence (also denoted by $\left\{u_{c}\right\}$ ) which converges weakly- $\star$ in $\mathcal{M}_{\Gamma}\left(\bar{\omega}_{c}\right)$ to a $\tilde{u} \in \mathcal{M}_{\Gamma}\left(\bar{\omega}_{c}\right)$. Since $S_{\omega}\left(u_{c}\right) \rightarrow S_{\omega}(\tilde{u})$ strongly in $L^{2}\left(\omega_{o}\right)$ by Proposition 2.1, we deduce from the continuity of $S_{\omega}^{*}$ that

$$
p_{\mathrm{c}} \rightarrow \tilde{\mathrm{p}}:=\mathrm{S}_{\omega}^{*}\left(\mathrm{~S}_{\omega} \tilde{\mathrm{u}}-z\right)
$$

strongly in $\mathrm{W}^{1, q^{\prime}}\left(\omega_{\mathrm{c}}\right)$ and hence in $\mathrm{C}_{\Gamma}\left(\bar{\omega}_{\mathrm{c}}\right)$.

We next verify the feasibility of $\tilde{p}$. By squaring the second relation of $\left(\mathrm{OS}_{\mathrm{c}}\right)$ and inspecting pointwise, we obtain that

$$
\frac{1}{c}\left\|u_{c}\right\|_{L^{2}\left(\omega_{c}\right)}^{2}=c\left\|\max \left(0, p_{c}-\alpha\right)\right\|_{L^{2}\left(\omega_{c}\right)}^{2}+c\left\|\min \left(0, p_{c}+\alpha\right)\right\|_{L^{2}\left(\omega_{c}\right)}^{2} .
$$

From (3.5), we have that $\frac{1}{c}\left\|u_{c}\right\|_{L^{2}\left(\omega_{c}\right)}^{2} \leqslant\|z\|_{L^{2}\left(\omega_{0}\right)}^{2}$, so that

$$
\begin{aligned}
& \left\|\max \left(0, p_{c}-\alpha\right)\right\|_{L^{2}\left(\omega_{c}\right)}^{2} \leqslant \frac{1}{c}\|z\|_{L^{2}\left(\omega_{0}\right)}^{2} \rightarrow 0, \\
& \left\|\min \left(0, p_{c}+\alpha\right)\right\|_{L^{2}\left(\omega_{c}\right)}^{2} \leqslant \frac{1}{c}\|z\|_{L^{2}\left(\omega_{o}\right)}^{2} \rightarrow 0,
\end{aligned}
$$


hold for $c \rightarrow \infty$. Since $p_{c} \rightarrow \tilde{p}$ strongly in $C_{\Gamma}\left(\bar{\omega}_{c}\right)$, this implies that

$$
-\alpha \leqslant \tilde{p}(x) \leqslant \alpha \quad \text { for all } x \in \bar{\omega}_{c} .
$$

It remains to pass to the limit in the second equation of $\left(\mathrm{OS}_{\mathrm{c}}\right)$. Observe that

$$
\begin{aligned}
\left\langle-u_{c}, p-p_{c}\right\rangle_{L^{2}\left(\omega_{c}\right)}= & c\left\langle\max \left(0, p_{c}-\alpha\right), p-p_{c}\right\rangle_{L^{2}\left(\omega_{c}\right)} \\
& +c\left\langle\min \left(0, p_{c}+\alpha\right), p-p_{c}\right\rangle_{L^{2}\left(\omega_{c}\right)} \leqslant 0
\end{aligned}
$$

holds for all $p \in \mathrm{C}_{\Gamma}\left(\bar{\omega}_{\mathrm{c}}\right)$ with $\|\mathrm{p}\|_{\mathrm{C}_{\Gamma}\left(\bar{\omega}_{\mathrm{c}}\right)} \leqslant \alpha$, and thus that

$$
\langle\tilde{u}, \tilde{p}-p\rangle_{\mathcal{M}_{\Gamma}\left(\bar{\omega}_{c}\right), C_{\Gamma}\left(\bar{\omega}_{c}\right)} \leqslant 0
$$

is satisfied for all $p \in \mathrm{C}_{\Gamma}\left(\overline{\boldsymbol{\omega}}_{\mathrm{c}}\right)$ with $\|p\|_{\mathrm{C}_{\Gamma}\left(\overline{\boldsymbol{w}}_{\mathrm{c}}\right)} \leqslant \alpha$. Therefore, $(\tilde{u}, \tilde{\mathrm{p}}) \in \mathcal{M}_{\Gamma}\left(\overline{\boldsymbol{\omega}}_{\mathrm{c}}\right) \times \mathrm{C}_{\Gamma}\left(\overline{\boldsymbol{\omega}}_{\mathrm{c}}\right)$ satisfies (OS).

Remark 3.3 (Non-negative controls). By a similar argument as in the proof of Theorem 3.1, it can be shown that

$$
\mathcal{G}_{+, c}^{*}: \mathrm{L}^{2}\left(\omega_{\mathrm{c}}\right) \rightarrow \overline{\mathbb{R}}, \quad v \mapsto \mathrm{I}_{\{v \leqslant 0\}}+\frac{1}{2 \mathrm{c}}\|v\|_{\mathrm{L}^{2}\left(\omega_{\mathrm{c}}\right)}^{2}+\alpha\|v\|_{\mathrm{L}^{1}\left(\omega_{\mathrm{c}}\right)},
$$

is the Fenchel conjugate of

$$
\mathcal{G}_{+, c}: \mathrm{L}^{2}\left(\omega_{\mathrm{c}}\right) \rightarrow \overline{\mathbb{R}}, \quad \mathrm{q} \mapsto \frac{\mathrm{c}}{2}\|\min (0, \mathrm{q}+\alpha)\|_{\mathrm{L}^{2}\left(\omega_{\mathrm{c}}\right)}^{2},
$$

and thus that the corresponding regularized optimality system is

$\left(\mathrm{OS}_{+, \mathrm{c}}\right)$

$$
\left\{\begin{aligned}
p_{c} & =S_{\omega}^{*}\left(S_{\omega} u_{c}-z\right), \\
-u_{c} & =c \min \left(0, p_{c}+\alpha\right) .
\end{aligned}\right.
$$

The convergence result for $c \rightarrow \infty$ as in Theorem 3.2 remains valid.

\section{SEMI-SMOOTH NEWTON METHOD}

For the numerical solution, we consider $\left(\mathrm{OS}_{c}\right)$ as the operator equation $F\left(u_{c}\right)=0$ for $F$ : $\mathrm{L}^{2}\left(\omega_{\mathrm{c}}\right) \rightarrow \mathrm{L}^{2}\left(\omega_{\mathrm{c}}\right)$, given by

$$
\mathrm{F}(\mathrm{u})=\mathrm{u}+\mathrm{c} \max \left(0, \mathrm{~S}_{\omega}^{*}\left(\mathrm{~S}_{\omega} \mathrm{u}-z\right)-\alpha\right)+\mathrm{c} \min \left(0, \mathrm{~S}_{\omega}^{*}\left(\mathrm{~S}_{\omega} u-z\right)+\alpha\right) .
$$

It is known (e.g., from [12, Ex. 8.14]), that the function $v \mapsto \max (0, v-\alpha)$ is Newton differentiable from $L^{p}$ to $L^{q}$ for any $p>q \geqslant 1$ with Newton derivative in direction $h$ given pointwise almost everywhere by

$$
\left[D_{N} \max (0, v-\alpha)\right] h=\chi_{\{v>\alpha\}} h= \begin{cases}h(x), & \text { if } v(x)>\alpha \\ 0, & \text { if } v(x) \leqslant \alpha\end{cases}
$$


An analogous statement holds for the pointwise min function. Since $S_{\omega}^{*} v \in \mathrm{W}^{1, \mathrm{q}^{\prime}}\left(\omega_{\mathrm{c}}\right)$ holds for all $v \in \mathrm{L}^{2}\left(\omega_{\mathrm{o}}\right), \mathrm{F}$ is Newton differentiable and the chain rule for Newton derivatives (e.g., [12, Lemma 8.15]) yields that the action of the Newton derivative of

$$
\mathrm{G}_{+}(\mathrm{u}):=\max \left(0, \mathrm{~S}_{\omega}^{*}\left(\mathrm{~S}_{\omega} \mathrm{u}-z\right)-\alpha\right)
$$

in the direction $h$ is given by

$$
\begin{aligned}
D_{N} G_{+}(u) h & =\chi_{\left\{S_{\omega}^{*}\left(S_{\omega} u-z\right)>\alpha\right\}}\left(S_{\omega}^{*} S_{\omega} h\right) \\
& = \begin{cases}\left(S_{\omega}^{*} S_{\omega} h\right)(x) & \text { if }\left(S_{\omega}^{*}\left(S_{\omega} u-z\right)\right)(x)>\alpha, \\
0 & \text { if }\left(S_{\omega}^{*}\left(S_{\omega} u-z\right)\right)(x) \leqslant \alpha,\end{cases}
\end{aligned}
$$

and a similar claim holds for the min term. A semi-smooth Newton step thus consists in solving for $\delta \mathrm{u}$ in the equation

$$
\begin{aligned}
\delta u+c \chi_{\left\{\left|S_{\omega}^{*}\left(S_{\omega} u^{k}-z\right)\right|>\alpha\right\}}\left(S_{\omega}^{*} S_{\omega} \delta u\right)=-u^{k} & -c \max \left(0, S_{\omega}^{*}\left(S_{\omega} u^{k}-z\right)-\alpha\right) \\
& -c \min \left(0, S_{\omega}^{*}\left(S_{\omega} u^{k}-z\right)+\alpha\right)
\end{aligned}
$$

and setting $u^{k+1}=u^{k}+\delta u$. The semi-smooth Newton step (4.1) can be solved using an iterative Krylov solver (e.g., GMREs), where the action of the Newton derivative on given $\delta u$ is computed by first solving the linearized state equation for the state differential $\delta y$ followed by the adjoint equation for the adjoint differential $\delta p$. The full procedure is given as Algorithm 1.

It remains to verify the well-posedness and uniform boundedness of the Newton step (4.1).

Proposition 4.1. For fixed $\alpha, c>0$ and for any $u \in \mathrm{L}^{2}\left(\omega_{c}\right)$, the mapping $\mathrm{D}_{\mathrm{N}} \mathrm{F}(\mathrm{u}) \in$ $\mathcal{L}\left(\mathrm{L}^{2}\left(\omega_{\mathrm{c}}\right), \mathrm{L}^{2}\left(\omega_{\mathrm{c}}\right)\right)$ is invertible, and there exists a constant $\mathrm{C}>0$ independent of $\mathrm{u}$ such that

$$
\left\|D_{N} F(u)^{-1}\right\|_{\mathcal{L}\left(L^{2}\left(\omega_{c}\right), L^{2}\left(\omega_{c}\right)\right)} \leqslant C
$$

holds.

Proof. Let $u \in \mathrm{L}^{2}\left(\omega_{\mathrm{c}}\right)$ be given and set

$$
\mathcal{A}=\left\{\left|S_{\omega}^{*}\left(S_{\omega} u-z\right)\right|>\alpha\right\}
$$

as well as $\mathcal{J}=\bar{\omega}_{\mathrm{c}} \backslash \mathcal{A}$.

For arbitrary $w \in \mathrm{L}^{2}\left(\omega_{\mathrm{c}}\right)$, we need to find $\delta u \in \mathrm{L}^{2}\left(\omega_{\mathrm{c}}\right)$ satisfying

$$
\delta u+c \chi_{\mathcal{A}}\left(S_{\omega}^{*} S_{\omega} \delta u\right)=w .
$$

From this, we have that $\delta u=w$ almost everywhere in J. By the linearity of $S_{\omega}$ and $S_{\omega}^{*}$, we can thus write

$$
c \chi_{\mathcal{A}}\left(S_{\omega}^{*} S_{\omega} \delta u\right)=c \chi_{\mathcal{A}}\left(S_{\omega}^{*} S_{\omega}\left(\chi_{\mathcal{A}} \delta u\right)\right)+c \chi_{\mathcal{A}}\left(S_{\omega}^{*} S_{\omega}\left(\chi_{\mathcal{J}} w\right)\right)
$$




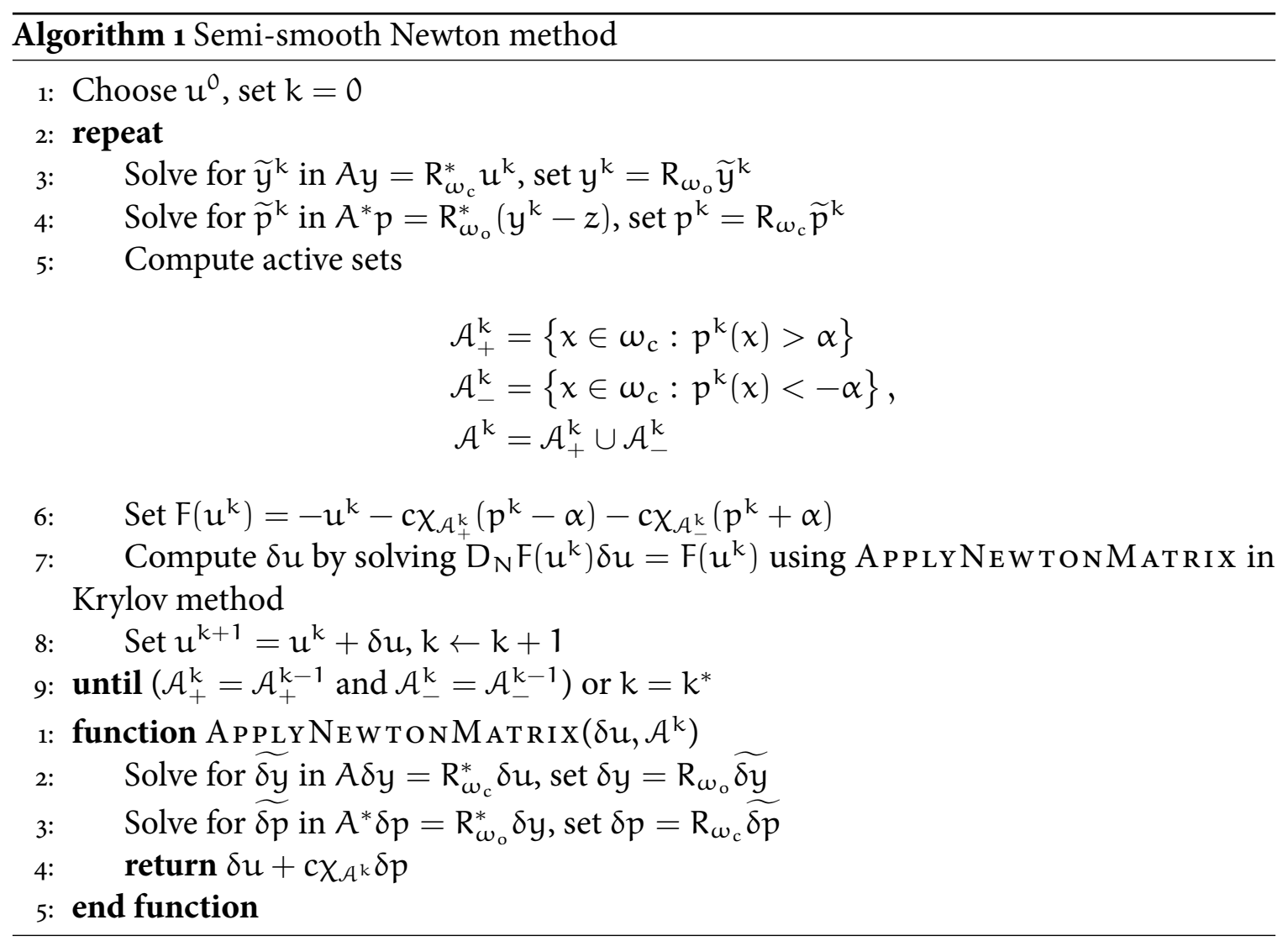

Inserting this identity into (4.2) and testing with $\delta \mathrm{u}$, we obtain

$$
\begin{aligned}
\|\delta \mathrm{u}\|_{\mathrm{L}^{2}\left(\omega_{\mathrm{c}}\right)}^{2}+\mathrm{c}\left\|\mathrm{S}_{\omega}\left(\chi_{\mathcal{A}} \delta \mathrm{u}\right)\right\|_{\mathrm{L}^{2}\left(\omega_{\mathrm{o}}\right)}^{2} & =\langle w, \delta \mathrm{u}\rangle_{\mathrm{L}^{2}\left(\omega_{\mathrm{c}}\right)}-\mathrm{c}\left\langle\mathrm{S}_{\omega}\left(\chi_{\mathcal{J}} w\right), \mathrm{S}_{\omega}\left(\chi_{\mathcal{A}} \delta \mathrm{u}\right)\right\rangle_{\mathrm{L}^{2}\left(\omega_{\mathrm{o}}\right)} \\
& \leqslant\|w\|_{\mathrm{L}^{2}\left(\omega_{\mathrm{c}}\right)}\|\delta \mathrm{u}\|_{\mathrm{L}^{2}\left(\omega_{\mathrm{c}}\right)}+\mathrm{C}\|w\|_{\mathrm{L}^{2}\left(\omega_{\mathrm{c}}\right)}\|\delta \mathrm{u}\|_{\mathrm{L}^{2}\left(\omega_{\mathrm{c}}\right)} \\
& \leqslant \mathrm{C}\|w\|_{\mathrm{L}^{2}\left(\omega_{\mathrm{c}}\right)}\|\delta \mathrm{u}\|_{\mathrm{L}^{2}\left(\omega_{\mathrm{c}}\right)}
\end{aligned}
$$

by the continuity of $S_{\omega}$. Together this implies

$$
\|\delta \mathrm{u}\|_{\mathrm{L}^{2}\left(\omega_{\mathrm{c}}\right)} \leqslant \mathrm{C}\|w\|_{\mathrm{L}^{2}\left(\omega_{\mathrm{c}}\right)}
$$

with a constant $\mathrm{C}>0$ depending on $\mathrm{c}$ but independent of $\mathcal{A}$ and therefore of $u$, which yields the claimed uniform boundedness.

From this, the superlinear convergence of the semi-smooth Newton method follows from standard arguments (e.g., [12, Th. 8.16]). The termination criterion in Algorithm 1, step 9, can be justified as follows: If $\mathcal{A}_{ \pm}^{k+1}=\mathcal{A}_{ \pm}^{k}$ holds, then $u^{k+1}$ satisfies $\mathrm{F}\left(\mathrm{u}^{\mathrm{k}+1}\right)=0$ (cf. [12, Rem. 7.1.1]).

For the numerical implementation, we use a continuation strategy: Solve for $u_{c_{k}}$, set $c_{k+1}=$ $q c_{k}$ with $q>1$, and use $u_{c_{k}}$ as initial guess for the computation of $u_{c_{k+1}}$.

Remark 4.2 (Non-negative controls). By setting $\mathcal{A}_{+}^{k}=\emptyset$, Algorithm 1 can be applied to the numerical solution of $\left(\mathrm{OS}_{+, \mathrm{c}}\right)$. The superlinear convergence holds in this case as well. 


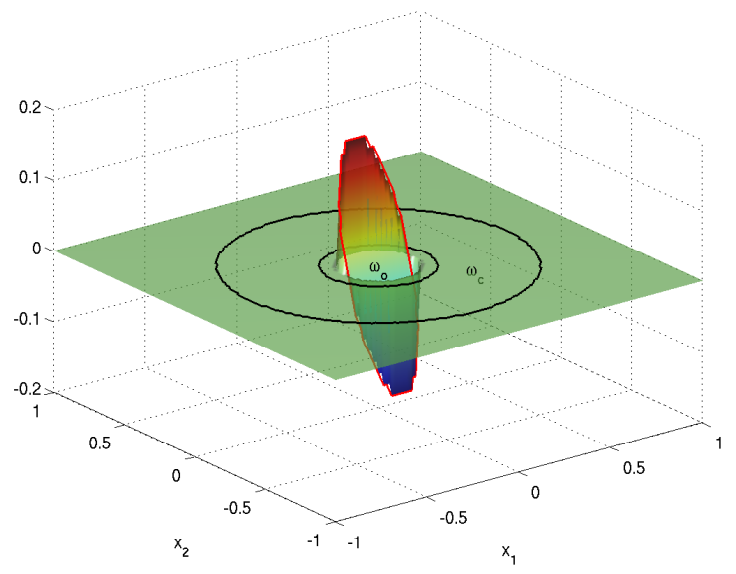

Figure 1: Target $z$, control domain $\omega_{\mathrm{c}}$ and observation domain $\omega_{\mathrm{o}}$.

\section{NUMERICAL EXAMPLES}

We illustrate the proposed approach with a simple convection-diffusion equation, described by the operator $A y=-v \Delta y-b \cdot \nabla y$ with $v=0.1$ and $b=(1,0)^{\top}$ and homogeneous Dirichlet conditions on the unit square $[-1,1]^{2}$. The control and observation domains are given by

$$
\begin{aligned}
& \omega_{c}=\left\{x \in \Omega: \frac{1}{16} \leqslant|x|^{2} \leqslant \frac{1}{2}\right\}, \\
& \omega_{\mathrm{o}}=\left\{x \in \Omega:|x|^{2} \leqslant \frac{1}{32}\right\},
\end{aligned}
$$

and the target is $z=\chi_{\omega_{0}} \chi_{2}$ (see Figure 1). The differential operators are discretized using standard finite differences with $\mathrm{N}=128$ nodes in each direction, and Algorithm 1 is implemented in MATLAB.

The parameters for this example are set as follows. In the continuation scheme for the penalty parameter $c$, the initial value is $c_{0}=1$, the incrementation factor is set to $q=10$, and the continuation is terminated at $c^{*}=10^{12}$. The semi-smooth Newton method is terminated if the active sets coincide or $k^{*}=10$ iterations are reached. For the solution of the linear systems arising in the semi-smooth Newton step, we use MAT L A B's built-in GMRES with a relative tolerance of $10^{-9}$ and a maximum number of iterations of 100. The MAT L A B code of our implementation can be downloaded from http : / /Www . uni - graz . at/ clason/codes/ measurecontrol.m.

The optimal controls $u_{\alpha}$ and corresponding states $y_{\alpha}$ for different values of $\alpha$ are shown in Figure 2. As $\alpha$ is decreased, the state becomes closer to the target, while the control becomes less sparse. Note that the loss in sparsity is manifested by an increasing number of point sources, but the support of the control remains localized. This is due to the structural properties of the optimality system: the control is allowed to be active only where the dual variable $p^{*}$ is active and must be identically zero everywhere else (cf. Corollary 2.4). Also, the controls are 
placed asymmetrically due to the directionality of the convection term. We point out that the placement of the corresponding sources is not obvious.

We indicate the superlinear convergence of the semi-smooth Newton method by fixing $c=10^{5}$ and $\alpha=10^{-4}$ and starting from the initial guess $u_{0}=0$. Table 1 shows the norm of the residual $\left\|\mathrm{F}\left(\mathrm{u}^{\mathrm{k}}\right)\right\|_{\mathrm{L}^{2}}$ and the change in active sets $\delta \mathcal{A}^{\mathrm{k}}$ for each iteration in the semi-smooth Newton method, verifying the locally superlinear convergence.

The feasibility of our approach for the optimal placement of sources is illustrated with an example that is motivated by an application in photochemotherapy. Here, $\omega_{\mathrm{o}}$ denotes a region where a photosensitive chemotherapeutic agent is locally activated by laser light from multiple strategically placed fiber-optic light sources [2]. The latter can be focused inside a small boundary layer, which corresponds to the control domain $\omega_{\mathrm{c}}$. This example further demonstrates the dependence of the locations of the optimal controls on the geometry of the problem, here determined by an irregular domain (see Figure 3).

The corresponding state equation is

$$
\left\{\begin{aligned}
-\nabla \cdot\left(\frac{1}{2\left(\mu_{\mathrm{a}}+\mu_{\mathrm{s}}\right)} \nabla y\right)+\mu_{\mathrm{a}} y & =\chi_{\omega_{\mathrm{c}}} \mathrm{u} & & \text { on } \Omega, \\
\frac{1}{2\left(\mu_{\mathrm{a}}+\mu_{\mathrm{s}}\right)} \partial_{v} y+\rho y & =0 & & \text { on } \partial \Omega,
\end{aligned}\right.
$$

which describes diffusive photon transport in tissue. Here, $\mu_{a}$ is the tissue's absorption coefficient, $\mu_{\mathrm{s}}$ is the scattering coefficient, and $\rho$ is the reflection coefficient at the boundary $\partial \Omega$. In our tests, we set $\mu_{a}=0.03, \mu_{s}=0.275$ and $\rho=0.1992$ to model a small piece of lung tissue. The objective is then to achieve a homogeneous illumination of the region of interest $\omega_{\mathrm{o}}$ for the optimal activation of the chemo-sensitive agent. Due to the linearity of the equation, we set without loss of generality $z \equiv 1$. As noted in Remark 4.2, non-negativity of the controls is enforced by setting $\mathcal{A}_{+} \equiv \emptyset$.

Due to the irregularity of the domain, we now use a standard finite element discretization of state and adjoint equation in weak form (cf. (2.2)) with triangular elements, where the discretized control is taken as piecewise constant and the discretized state and adjoint as piecewise linear. The implementation is based on the open source FENICS project [13]. Here, the final penalty parameter is set to $c^{*}=10^{9}$, the remaining parameters being unchanged. The results for different values of $\alpha$ are shown in Figure 4. The influence of $\alpha$ on sparsity of the controls and homogeneity of the illumination can be observed clearly. Note that the placement of the optimal point sources is again not obvious, and depends on $\alpha$ in a non-intuitive manner

Table 1: Convergence of semi-smooth Newton method. Shown are the norm of the residual of (4.1) and the change in active sets $\delta \mathcal{A}^{\mathrm{k}}$ in each iteration.

\begin{tabular}{cccccc}
\hline $\mathrm{k}$ & 1 & 2 & 3 & 4 & 5 \\
\hline$\left\|\mathrm{F}\left(\mathrm{u}^{\mathrm{k}}\right)\right\|$ & $3.84 \cdot 10^{2}$ & $3.78 \cdot 10^{1}$ & $6.12 \cdot 10^{0}$ & $6.16 .78 \cdot 10^{-1}$ & $1.08 \cdot 10^{-10}$ \\
$\delta \mathcal{A}^{\mathrm{k}}$ & 3678 & 532 & 106 & 8 & 0 \\
\hline
\end{tabular}




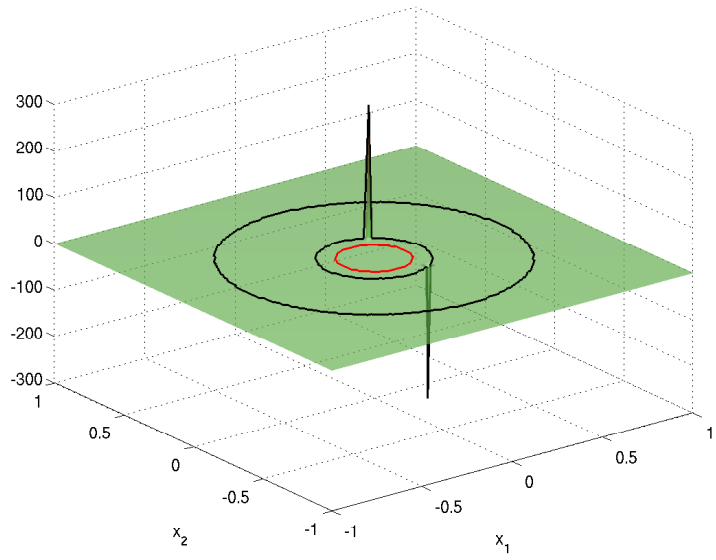

(a) $\mathfrak{u}_{\alpha}, \alpha=10^{-3}$

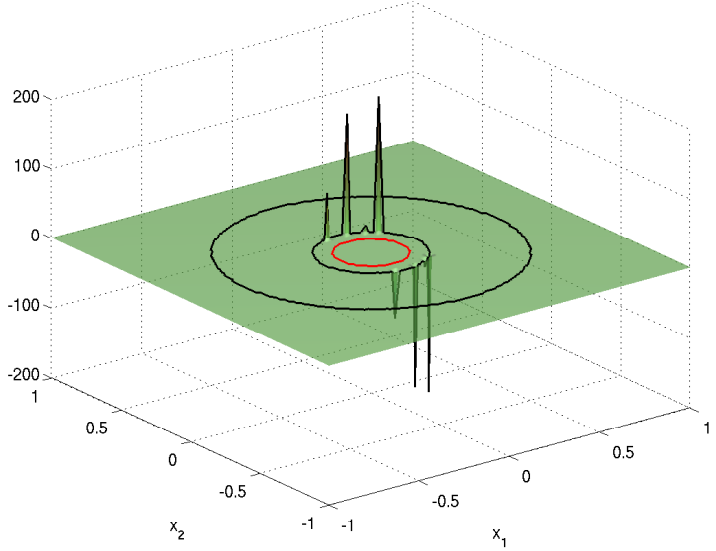

(c) $u_{\alpha}, \alpha=10^{-4}$

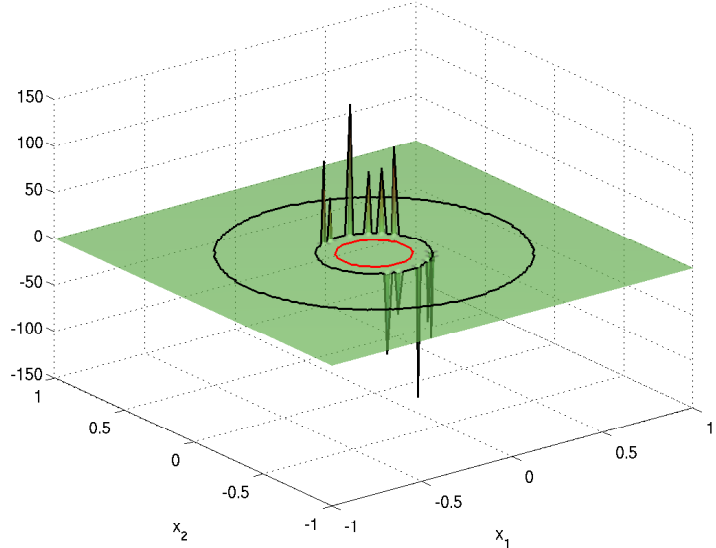

(e) $u_{\alpha}, \alpha=10^{-5}$

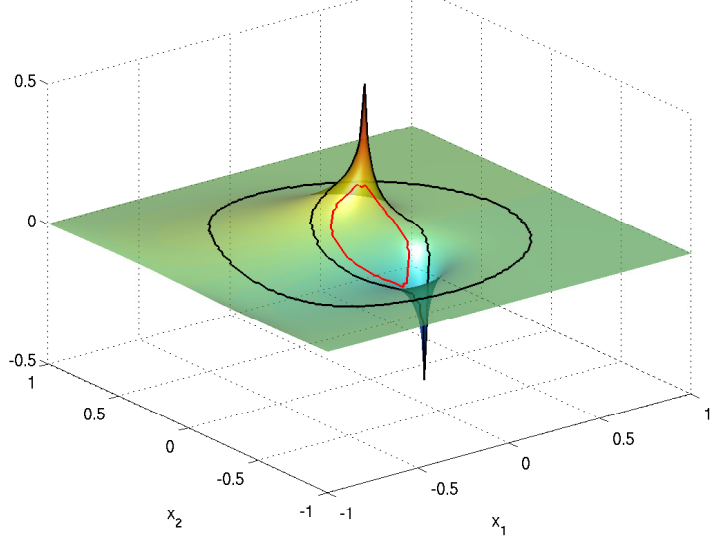

(b) $y_{\alpha}, \alpha=10^{-3}$

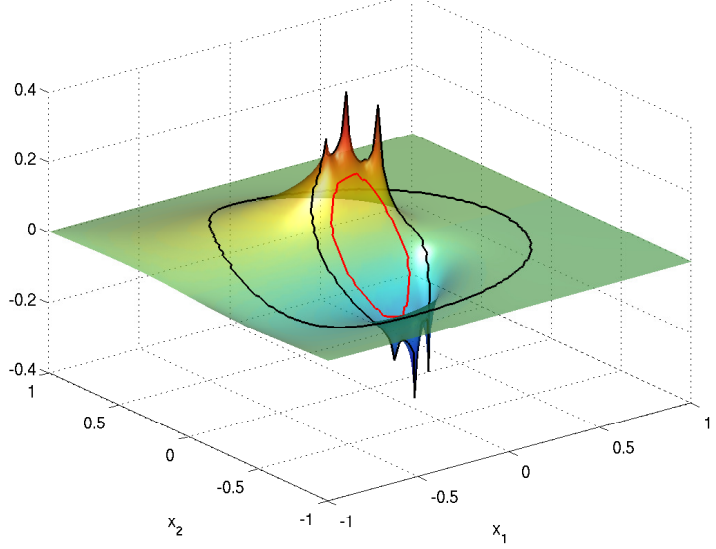

(d) $y_{\alpha}, \alpha=10^{-4}$

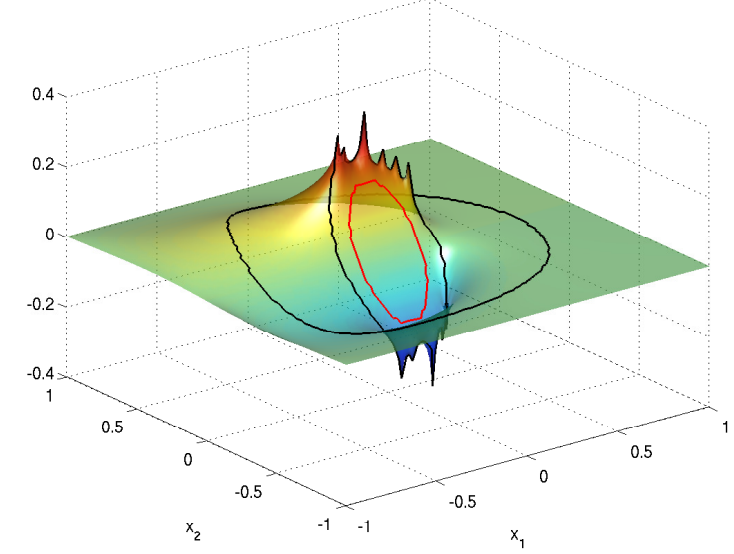

(f) $y_{\alpha}, \alpha=10^{-5}$

Figure 2: Optimal controls $u_{\alpha}$ and states $y_{\alpha}$ for different values of $\alpha$. 


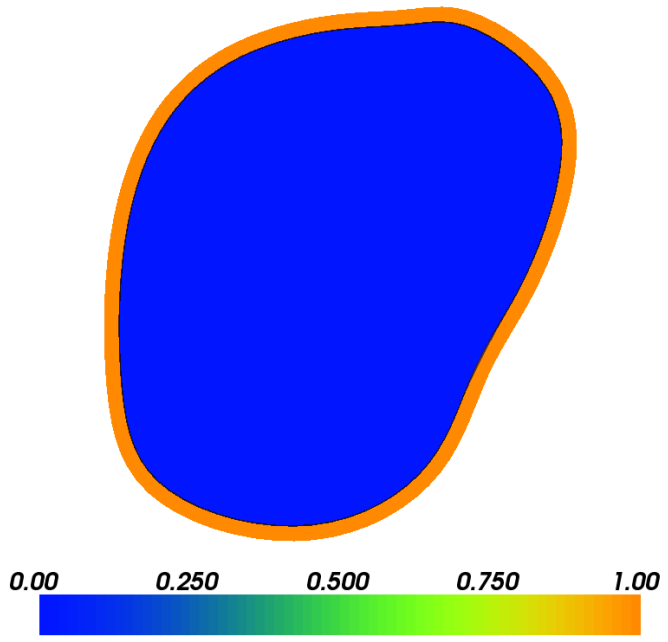

(a) control domain $\omega_{c}$

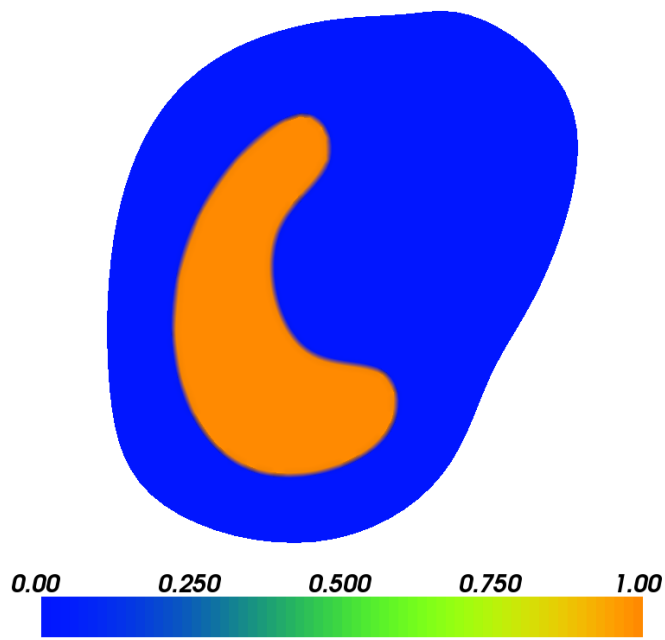

(b) observation domain $\omega_{\mathrm{o}}$

Figure 3: Geometry for model problem. Shown are the indicator functions of control and observation domain.

(compare the location of the major point source on the right hand side of the domain between Figs. $4 \mathrm{a}$ and $4 \mathrm{~b})$.

\section{CONCLUSION}

The problem of optimal placement of point sources was formulated in a non-reflexive Banach space setting. The optimality system for this non-smooth optimization problem was derived and a family of regularized problems, which can be approximated efficiently by semi-smooth Newton methods, was analyzed. The numerical examples demonstrate the effectivity for optimal light source placement problems in diffusive photochemotherapy. Current work is concerned with the application of the proposed approach to patient-specific geometries. Formally, the primal-dual framework considered here can be extended to nonlinear controlto-state mappings, although the proper functional analytic treatment of the linearization remains challenging. Finally, it would be of interest to consider parabolic state equations.

\section{ACKNOWLEDGMENTS}

The authors wish to thank Patricia Brunner, Manuel Freiberger and Hermann Scharfetter of the Institute of Medical Engineering, Graz University of Technology, for their help on the photochemotherapy example. 


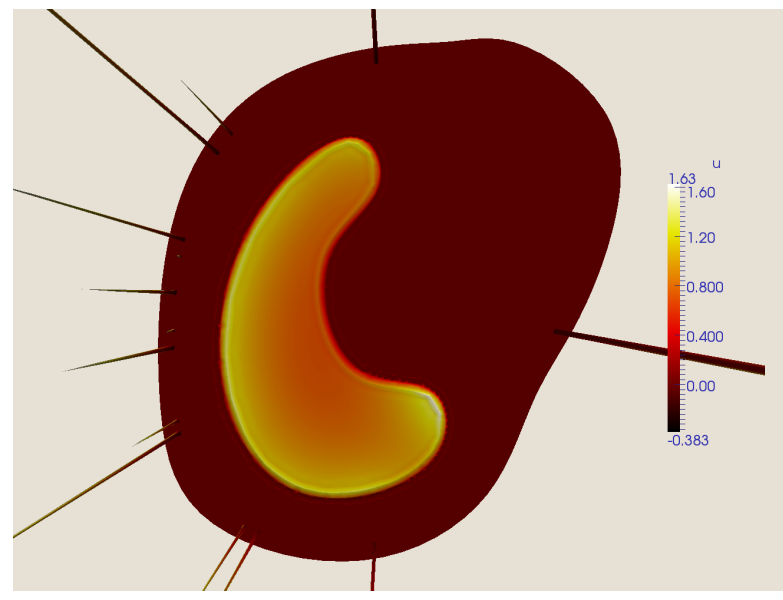

(a) $\alpha=5 \cdot 10^{-2}$

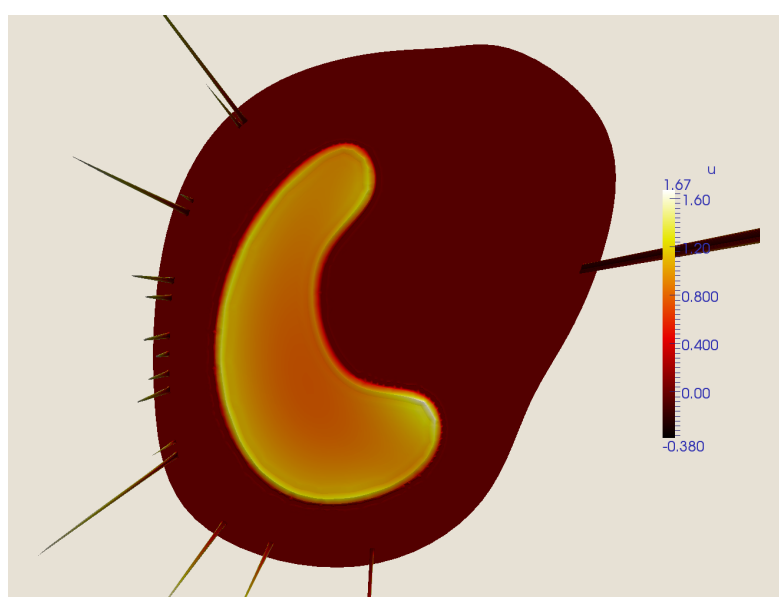

(c) $\alpha=5 \cdot 10^{-3}$

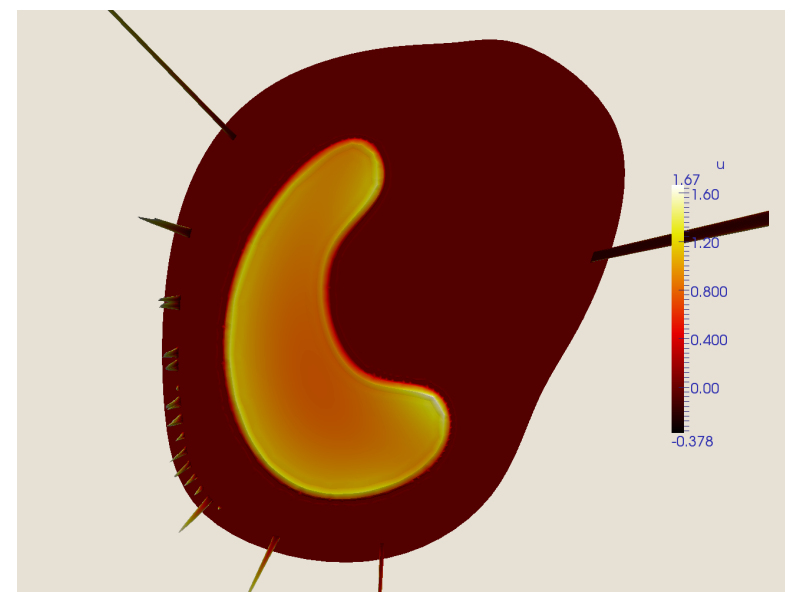

(e) $\alpha=5 \cdot 10^{-4}$

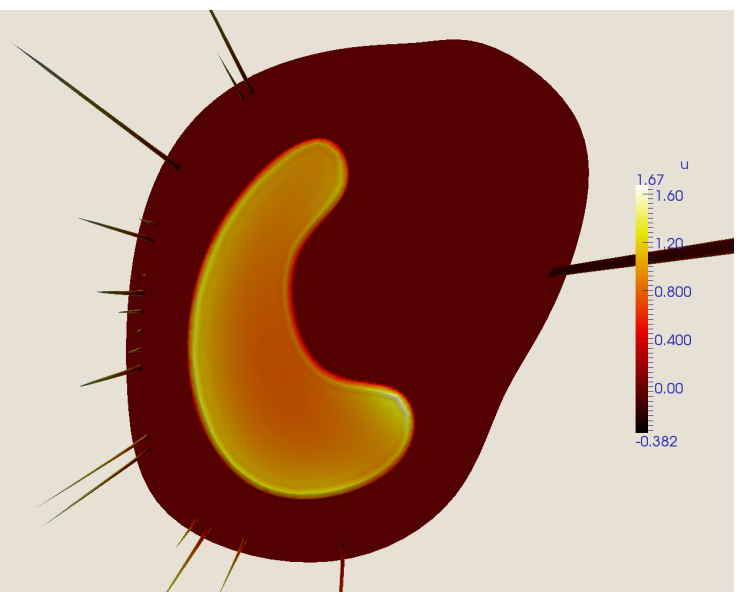

(b) $\alpha=1 \cdot 10^{-2}$

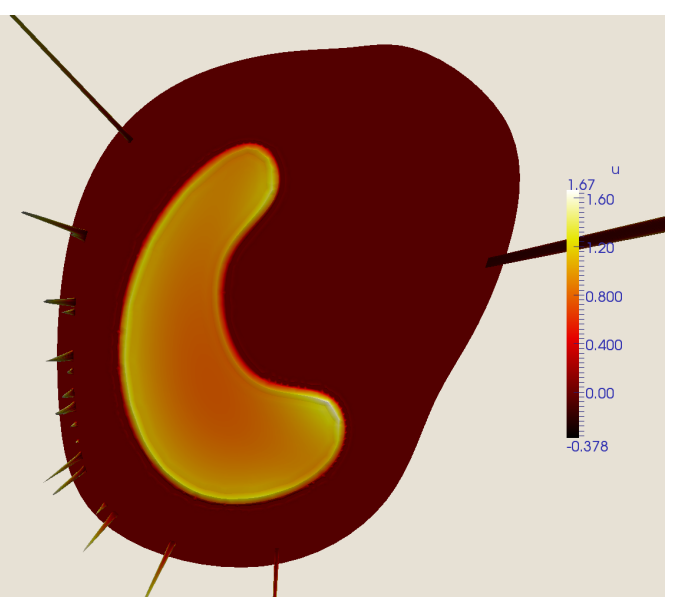

(d) $\alpha=1 \cdot 10^{-3}$

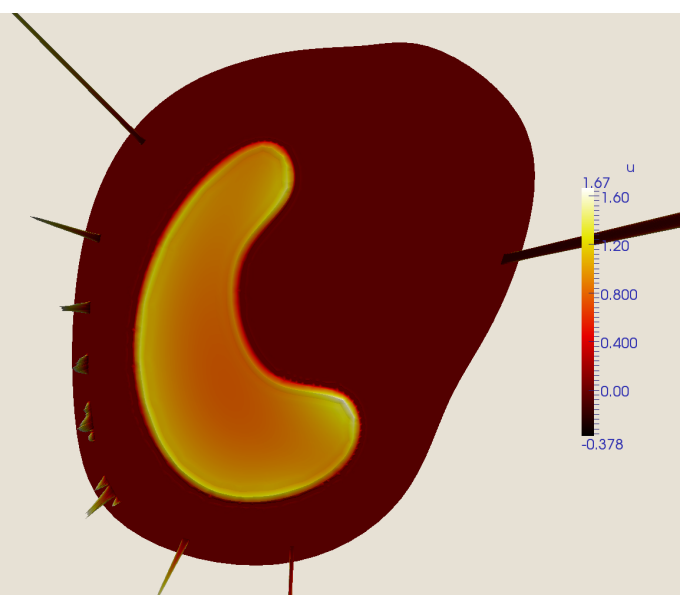

(f) $\alpha=1 \cdot 10^{-4}$

Figure 4: Optimal controls $u$ and state $y$ for different values of $\alpha$. Shown is a superposition of $u$ on $\omega_{c}$ (as height plot) and $y$ on $\omega_{o}$ (as color plot). 


\section{REFERENCES}

[1] H. Attouch, G. Buttazzo, and G. Michaille, Variational Analysis in Sobolev and BV Spaces, Society for Industrial and Applied Mathematics (SIAM), Philadelphia, PA, 2006.

[2] P. Baas, L. Murrer, F. Zoetmulder, F. Stewart, H. Ris, N. van Zandwijk, J. Peterse, And E. Rut gers, Photodynamic therapy as adjuvant therapy in surgically treated pleural malignancies, Br. J. Cancer, 76 (1997), pp. 819-826.

[3] K. BREDIES AND H. PIKKARAINEN, Inverse problems in spaces of measures, Tech. Rep. 2010-037, Mobis, 2010.

[4] H. Brezis, Functional Analysis, Sobolev Spaces and Partial Differential Equations, Springer, New York, 2010.

[5] E. Casas, R. Herzog, and G. Wachsmuth, Optimality conditions and error analysis of semilinear elliptic control problems with $\mathrm{L}^{1}$ cost functional, 2011. Submitted.

[6] C. Clason and K. Kunis CH, A duality-based approach to elliptic control problems in non-reflexive Banach spaces, ESAIM: Control, Optimisation and Calculus of Variations, 17 (2011), pp. 243-266.

[7] E. DiBenedet to, Real Analysis, Birkhäuser Boston Inc., Boston, MA, 2002.

[8] I. Ekela nd and R. Tém a m, Convex Analysis and Variational Problems, Society for Industrial and Applied Mathematics, Philadelphia, PA, USA, 1999.

[9] J. Els trodt, Maß- und Integrationstheorie, Springer-Verlag, Berlin, 5 ed., 2005.

[10] T. Gallouet and A. Monie R, On the regularity of solutions to elliptic equations, Rend. Mat. Appl. (7), 19 (1999), pp. 471-488 (2000).

[11] M. HintermülleR AND K. KunisCH, Total bounded variation regularization as a bilaterally constrained optimization problem, SIAM J. Appl. Math., 64 (2004), pp. 1311-1333 (electronic).

[12] K. Ito And K. Kunisch, Lagrange Multiplier Approach to Variational Problems and Applications, vol. 15 of Advances in Design and Control, Society for Industrial and Applied Mathematics (SIAM), Philadelphia, PA, 2008.

[13] A. Logg And G. N. Wells, DOLFIN: Automated finite element computing, ACM Trans. Math. Softw., 37 (2010), pp. 20:1-20:28.

[14] C. Meyer, L. PANizzi, And A. SChiela, Uniqueness criteria for solutions of the adjoint equation in state-constrained optimal control, Numerical Functional Analysis and Optimization, (2010). To appear. 
[15] G. STADLE R, Elliptic optimal control problems with $\mathrm{L}^{1}$-control cost and applications for the placement of control devices, Computational Optimization and Applications, 44 (2009), pp. 159-181.

[16] G. Sta mpacchi a, Le problème de Dirichlet pour les équations elliptiques du second ordre à coefficients discontinus, Ann. Inst. Fourier (Grenoble), 15 (1965), pp. 189-258.

[17] G. M. Troin iello, Elliptic Differential Equations and Obstacle Problems, The University Series in Mathematics, Plenum Press, New York, 1987.

[18] D. Wachsmuth and G. Wachsmuth, Convergence and regularization results for optimal control problems with sparsity functional, ESAIM: Control, Optimisation and Calculus of Variations, (2010).

[19] - On the regularization of optimization problems with inequality constraints, Tech. Rep. 2011-1, RICAM Report, 2011. 